\title{
ACTITUD ALIMENTARIA ANÓMALA Y SU RELACIÓN CON LA IMAGEN CORPORAL EN UNA MUESTRA URBANA DE ADOLESCENTES VALENCIANOS.
}

\author{
ANA SÁNCHEZ ${ }^{1}$, FELIPE HURTADO ${ }^{2}$, JULIA COLOMER ${ }^{3}$, SILVIA SARAVIA ${ }^{3}$, \\ JOSÉ MONLEÓN ${ }^{4}$ y AMPARO SORIANO ${ }^{4}$ \\ ${ }^{1}$ Unidad de Salud Mental Fuente San Luis de Valencia, ${ }^{2}$ Planificación Familiar Fuente San Luis de Valencia, \\ ${ }^{3}$ Departamento de Pediatría, Universidad de Valencia, ${ }^{4}$ Centro de Salud Fuente San Luis de Valencia
}

(Aceptado el 20 de octubre de 2000)

Los objetivos de este estudio fueron determinar la prevalencia de comportamientos alimentarios anómalos en una muestra de adolescentes de 14 a 18 años, de centros de enseñanza secundaria ubicados en el área de salud $n^{2} 9$ de Valencia y analizar la relación de dichos comportamientos con variables antropométricas, con la autoimagen corporal y con dietas. Se utilizó el Test de Actitudes Alimentarias (EAT) de Garner y Garfinkel, el Body Shape Questionnaire de Cooper. Los sujetos fueron pesados y tallados hallándose el índice de Quetelet, se recogió información sobre dietas, auto-valoración del volumen corporal y deseo de mantener su peso, disminuirlo o aumentarlo. La prevalencia de riesgo de sufrir un trastorno de la alimentación, fue del $19,8 \%$ en las mujeres $(n=197)$ y del $1,76 \%$ en los hombres $(n=170)$. Puntuaron significativamente más alto en el EAT, las mujeres de 16 años, los sujetos que desearon estar más delgados, las mujeres que se auto-valoraron con sobrepeso u obesidad, las que realizaron dieta durante el último año y conocían familiares y/o amigos haciéndola. La distorsión de la imagen corporal fue significativamente superior en las mujeres con sobrepeso objetivo, las que se auto-valoraban con problemas de peso, las que deseaban estar más delgadas y las que hacían dieta.

Palabras clave: Anorexia y bulimia nerviosas, trastornos de la alimentación, prevalencia, imagen corporal.

\section{Abnormal eating attitude and its relationship with body image in an urban sample of adolescents from Valencia}

The aims of the present research were to asses the prevalence of abnormal eating attitudes in a sample of adolescents, aged 14-18, who attend secondary school in the $9^{\text {th }}$ wealth area of Valencia, and analyse the relationship between such attitudes and anthropometric measurements, body self-image and diets. The Eating Attitudes Test (EAT) by Garner and Garfinkel, and the Body Shape Questionnaire by Cooper were used. The adolescents' weigh and eight were measured and the Quetelet Index was calculated. Information about diets, body shape self-evaluation and wish for changing weight was collected. Prevalence of risky eating attitudes, was $19.8 \%$ for the girls $(\mathrm{n}=197)$ and $1.76 \%$ for the boys $(n=170)$. The EAT scores were significantly higher for girls aged 16 , the subjects who wished a body shape reduction, women who self-evaluated with overweight and obesity, girls who were on a diet during the last year and women that knew fiends and relatives going on it. The distortion of body shape was significantly higher for woman with objective overweight, women self-evaluated with overweight and obesity, women who wished a body shape reduction and women who were on a diet.

Key words: Anorexia and bulimia nervosa, eating disorder, prevalence, body shape.

Agradecimientos: A los Institutos Fuente San Luis, San Jordi, Salesianos y Tirant lo Blanc de Valencia por facilitar la recogida de información de sus alum. nos. A la Unidad Docente de Medicina Familiar y Comunitaria de Valencia por su apoyo metodológico en esta investigación.

Correspondencia: Ana Sánchez, Unidad de Salud Mental Fuente San Luis, c/ Arabista Ambrosio Huici 30, 46013 Valencia, Tfno.: 963745 795, Fax: 963732369 , Correo-e: edyana@cop.es 


\section{INTRODUCCIÓN}

En los últimos años ha aumentado el interés por los trastornos de la conducta alimentaria especialmente, anorexia y bulimia nerviosa, tanto entre los profesionales implicados en su estudio y tratamiento como en la sociedad general.

En la década anterior el número de publicaciones era reducido, teniendo los profesionales dedicados al tratamiento de estos problemas, prácticamente como únicas lecturas de referencia en castellano, el libro sobre anorexia de Toro (Toro et al., 1987) y un capítulo con el mismo título de Calvo (Calvo, 1985) siendo aún más infrecuentes las publicaciones sobre bulimia nerviosa.

En la actualidad los medios de comunicación se ocupan cada vez más de estas patologías, el congreso de diputados ha propuesto leyes que inciden en la prevención de las mismas y se han generado recursos específicos para el tratamiento de estos trastornos, tales como unidades de hospitalización y hospitales de día.

Las investigaciones sobre el tema han proliferado, encontrando un incremento en la prevalencia de estos trastornos y cuestionando algunas de las premisas de las que se partía inicialmente, como el predominio en sujetos con rendimiento académico alto (Canals et al., 1996) o entre la clase social alta (Toro et al., 1989; Fisher et al., 1994; Gard et al., 1996). Al mismo tiempo se han desarrollado instrumentos de medida y una metodología de estudio que se están utilizando en diversos países del mundo occidental.

En los estudios epidemiológicos se han detectado como factores frecuentemente implicados en el desarrollo de los trastornos de la alimentación, entre otros, la edad, el sexo femenino, el sobrepeso y la percepción negativa de la imagen corporal con tendencia a hacer depender la autoestima del peso y la figura (Buddeberg-Fischer et al., 1996; Fairburn et al., 1997; Toro et al., 1994).
Con el presente trabajo nos proponemos: (1) determinar la prevalencia de comportamientos alimentarios anómalos, anorexia y bulimia nerviosa, en población de 14 a 18 años escolarizada, y (2) analizar la relación de comportamientos alimentarios anómalos con medidas antropométricas, edad, sexo, dietas y con la autoimagen corporal.

\section{MÉTODO}

\section{Diseño}

Se trata de un estudio descriptivo de prevalencia con componentes analíticos.

\section{Participantes}

Alumnos de enseñanzas medias seleccionados de dos institutos de Bachillerato y de un Instituto de Formación Profesional pertenecientes al sistema público de enseñanza y de un centro de EGB y BUP concertado, todos situados en el área 9 de salud de Valencia, zonas 03 y 02. Las edades comprenden un rango entre los 14 y 18 años correspondientes a las enseñanzas de $3 .^{\circ}$ y $4 .^{9}$ de ESO y Bachiller, en el nuevo sistema previsto en la LOGSE, BUP y COU, FP1 y FP2 en el antiguo modelo de enseñanza.

Se calculó un tamaño muestral de $\mathbf{5 1 2}$ sujetos para una prevalencia media de trastornos de la alimentación del $10 \%$, estimada a partir de la bibliografía revisada (Buddeberg-Fischer et al., 1996; Canals et al., 1996; Toro et al., 1989) para adolescentes sin distinción de sexo y mediante el uso de cuestionarios, con una precisión del $2 \%$ y una $\mathrm{a}=0,05$.

La selección de la muestra fue aleatoria por conglomerado, muestreándose las clases y de cada clase se estudió la totalidad de alumnos.

Se calculó una media de 30 alumnos por aula, seleccionando 22 aulas median- 
te muestreo aleatorio sistemático con una $\mathrm{K}=3$. Previamente se comprobó el número de aulas y alumnos por estrato (edad) para determinar la homogeneidad de la muestra.

\section{Variables}

- Variables demográficas: Edad, sexo y curso escolar.

- Variables antropométricas: Peso, altura, valorando índice de masa corporal (IMC) o índice de Quetelet, obtenido calculando el valor del cociente entre el peso (en Kg.) y la talla (en metros) elevada al cuadrado, según la fórmula: $\mathrm{IMC}=$ Peso $(\mathrm{Kg}$.$) / talla (\mathrm{m})^{2} . \mathrm{El}$ valor obtenido se comparó con las curvas estándar de población infantil de la Fundación Orbegozo, publicadas por Hernández en 1988 (Hernández et al., 1988). Se definió el estado nutricional en porcentaje del IMC ideal de un sujeto de su misma edad y sexo con peso y talla en percentil $(\mathrm{Pc})$ 50: IMC del sujeto a estudio / IMC en Pc.50 para su edad y sexo x 100 . Para clasificar el estado nutricional según este índice se aplicaron los valores de McLaren y Read (McLaren et al., 1972) por estar ampliamente difundidos en la literatura práctica relacionada con la nutrición. Así, se clasificaron a los sujetos en cuatro grupos, bajo peso o malnutrición (IMC inferior o igual al $90 \%$ del esperable, percentil 50, según su edad y sexo), normopeso o normonutrido (IMC 91-110), sobrepeso (IMC 111-120), y obesidad (IMC superior a 120) (Pombo, 1990). Se utilizó como criterios antropométricos de anorexia los establecidos por la Organización Mundial de la Salud en 1993 (CIE 10) (World Health Organization, 1993) (IMC igual o inferior a 17,5) y los propuestos por la Asociación de Psiquiatría America- na en 1994 (DSM IV) (A.P.A., 1994) (peso inferior al $85 \%$ del esperable porcentaje del IMC ideal de un sujeto de su misma edad y sexo con peso y talla en percentil (Pc) 50: IMC del sujeto a estudio / IMC en Pc.50 para su edad y sexo $\times 100$ ).

- Variables relacionadas con la alimentación y la imagen: Imagen corporal, actitudes y comportamientos alimentarios anómalos, dieta personal en el último año, dieta de familiares y/o amigos en el ultimo año, auto-valoración del volumen corporal y deseo de modificarlo.

\section{Instrumentos de medida}

Para las variables antropométricas se utilizó una báscula tipo romana marca Seca con precisión de \pm 100 gr. Talla acoplada sobre barra vertical de la báscula y con precisión de $\pm 1 \mathrm{~mm}$.

Test de Actitudes Alimentarias (EAT40), de Garner y Garfinkel (Garner et al., 1979), instrumento de autoinforme de 40 items que evalúa actitudes y comportamientos alimentarios anómalos, conteniendo como subescalas, 1) Bulimia y preocupación por la comida, 2) Seguimiento de dietas, y 3) Control oral. Cada ítem se contesta en una escala likert de 6 niveles de respuesta que van de "nunca» hasta «siempre». El punto de corte a partir del cual se considera riesgo patológico es de 30 y las puntuaciones globales de este cuestionario van de 0 a 120. Este test se ha utilizado ampliamente en estudios epidemiológicos y es reconocida su eficacia para detectar riesgo de padecer anorexia y bulimia nerviosa.

Body Shape Questionnaire (BSQ) para mujeres, de Cooper (Cooper et al., 1987) o "cuestionario de la forma corporal». Consta de 34 items que pueden contestarse en una escala likert de 6 niveles de respuesta, que van de "nunca» hasta 
«siempre». Mide insatisfacción corporal, miedo a engordar, sentimientos de baja autoestima a causa de la apariencia y deseo de perder peso. Muestra una consistencia interna de 0,93 y una buena validez concurrente con la subescala de «Insatisfacción corporal» del Inventario de Trastornos de la Alimentación (EDI). La fiabilidad y validez de este cuestionario ha sido estudiada por Rosen (Rosen $e t$ al., 1996), habiéndose utilizado en este estudio la traducción y adaptación de Mora y Raich (1993) (Mora et al., 1993)

Un protocolo de evaluación estructurado basado en el utilizado por Toro y colaboradores (Toro et al., 1989) recogiendo dieta llevada durante el último año, por los alumnos, sus familias y amigos; auto-valoración del volumen corporal (muy obeso, bastante obeso, un poco obeso, estoy normal, un poco delgado, bastante delgado y muy delgado); deseo de modificar el volumen corporal (más delgado, igual, más gordo).

\section{Recogida y análisis de datos}

El cuestionario BSQ para mujeres, el autoinforme EAT, así como el cuestionario construido a partir del utilizado por Toro y colaboradores, se administraron en formato de grupo por curso y aula, informando previamente a los alumnos de la confidencialidad de los datos y de que se trataba de un estudio para valorar su comportamiento y actitud en relación a la alimentación y a la imagen corporal.

Al completar el alumno sus respuestas, pasaba con sus cuestionarios a un espacio habilitado al efecto donde el examinador, con el sujeto descalzo y retirada la ropa de abrigo, pesaba y tallaba al alumno para, posteriormente, calcular el IMC y agrupar a los sujetos según los percentiles de este valor y clasificarlos según su estado nutricional.
Para elaborar la información recogida se diseñó una base de datos informatizada. Se realizó un análisis descriptivo de la muestra así como de las diferencias en función de las variables de agrupamiento, edad, sexo e IMC, utilizándose la t de Student para comparar las medias, chi cuadrado para comparar proporciones y el análisis de varianza, para comparar más de dos grupos. Se calculó la prevalencia de las conductas alimentarias anómalas, anorexia y bulimia nerviosas, con sus intervalos de confianza al $95 \%$. Se realizó análisis de regresión lineal para la relación EAT-BSQ.

\section{RESULTADOS}

Del total de alumnos que cumplimentaron la información (447), se eliminaron todos aquellos a los que les falto alguno de los items de los cuestionarios utilizados en el estudio y aquellos con edades superiores a los 18 años. De este modo el análisis estadístico se realizó sobre una muestra de 367 sujetos.

\section{Descripción demográfica y antropométrica}

De los 367 sujetos, el $46,3 \%$ fueron hombres y el $53,7 \%$ mujeres, no existiendo diferencias en la distribución por sexos entre los distintos grupos de edad. La edad media fue de 16,03 $(\mathrm{DT}=1,25)$ para los hombres y de 16,24 (DT $=1,19$ ) para las mujeres. No hubo diferencias estadísticas entre los sexos en la distribución por medidas antropométricas, ni siguiendo los percentiles del IMC, ni según los criterios para valorar su estado nutricional, ni en la distribución por sexo de los sujetos que cumplían criterios antropométricos de anorexia según DSM IV y CIE 10. En relación al índice de masa corporal 
(IMC), un $24,52 \%$ se situaron dos DS por encima de la media (percentil 90), mientras que los situados dos DS por debajo (percentil 10) fueron un 3,81\%, y entre una DS por encima y por debajo se encontraban un $46,32 \%$ de los sujetos (percentil 25 a 75). Según los criterios para establecer el estado nutricional, el $32,15 \%$ presentaron sobrepeso $u$ obesidad, frente a el $54,77 \%$ con normopeso y el $13,08 \%$ de bajo peso, no hallándose diferencias significativas ni por edad ni por sexo. La proporción de sujetos que cumplían con los criterios antropométricos de anorexia seguidos por DSM IV y CIE 10, fue de 3,5\% en el primero y de 3,3\% en el segundo, no observándose diferencias significativas ni por edades ni por sexo y tampoco entre ambos criterios (véase la Tabla 1).

\section{Relación EAT y BSQ con variables sociodemográficas}

Cuarenta y dos alumnos $(11,38 \%$ del total alumnos), superaron el punto de corte de 30 en el cuestionario EAT, de los que 39 fueron mujeres $(19,8 \%$ del total de mujeres) y 3 hombres (1,76\% del total de los hombres). La media obtenida por las mujeres en el cuestionario EAT fue significativamente más elevada que la de los hombres $(p<0,001)$. Las mujeres de 16 años puntuaron significativamente más alto que las mujeres de 17 y 18 años ( $p<0,001)$. En el cuestionario BSQ no se observaron diferencias significativas entre los diferentes grupos de edad, siendo la media global de 88, 37 y DS 37,85 (véase la Tabla 2).

\section{Relación entre el EAT y el BSQ}

El análisis de regresión lineal demos-

Tabla 1. Descripción de la muestra: variables demográficas y antropométricas

\begin{tabular}{|c|c|c|c|c|}
\hline Variables & $\begin{array}{l}\text { Hombres } \\
N \quad(\%)\end{array}$ & $\begin{array}{l}\text { Mujeres } \\
N \quad(\%)\end{array}$ & N Total & $\%$ del total \\
\hline \multicolumn{5}{|l|}{ Distribución por edad } \\
\hline$x^{2}$ & $25(57,89)$ & $16(42,11)$ & 38 & 10,35 \\
\hline 15 & $39(48,15)$ & $42(51,85)$ & 81 & 22,07 \\
\hline 16 & $45(47,87)$ & $49(52,13)$ & 94 & 25,61 \\
\hline 17 & $42(40,78)$ & $61(59,22)$ & 103 & 28,07 \\
\hline 18 & $22(43,14)$ & $29(56,86)$ & 51 & 13,90 \\
\hline Global & $170(46,32)$ & $197(53,68)$ & 367 & 100,00 \\
\hline \multicolumn{5}{|l|}{ Distribución por percentiles del IMC } \\
\hline$<3$ & $1 \quad(20,00)$ & $4 \quad(80,00)$ & 5 & 1,36 \\
\hline 4-10 & $3(33,33)$ & $6(66,67)$ & $\mathbf{9}$ & 2,45 \\
\hline $11-25$ & $22(43,14)$ & $29(56,86)$ & 51 & 13,90 \\
\hline $26-50$ & $51(53,13)$ & $45(46,88)$ & 96 & 26,16 \\
\hline $51-75$ & $36(48,65)$ & $38(51,35)$ & 74 & 20,16 \\
\hline $76-90$ & $24(57,14)$ & $18(42,86)$ & 42 & 11,44 \\
\hline $91-97$ & $7 \quad(20,59)$ & $27(79,41)$ & 34 & 9,26 \\
\hline$>97$ & $26(46,43)$ & $30(53,57)$ & 56 & 15,26 \\
\hline \multicolumn{5}{|c|}{ Evaluación del estado nutricional según \% sobre percentil 50 del IMC } \\
\hline Bajo peso $(<=90 \%)$ & $23(47,92)$ & $25(52,08)$ & 48 & 13,08 \\
\hline Normopeso (91-110\%) & $91(45,27)$ & $110(54,73)$ & 201 & $\mathbf{5 4 , 7 7}$ \\
\hline Sobrepeso $(111-120 \%)$ & $23(46,00)$ & $27(54,00)$ & 50 & 13,62 \\
\hline Obesidad ( $>120 \%)$ & $33(48,53)$ & $35(51,47)$ & 68 & 18,53 \\
\hline \multicolumn{5}{|c|}{ Sujetos con criterios antropométricos de anorexia } \\
\hline DSM IV (peso <85\%) & $6(46,15)$ & $7(53,85)$ & 13 & 3,54 \\
\hline CIE 10 (IMC <17,5\%) & $6(50,00)$ & $6(50,00)$ & 12 & 3,27 \\
\hline
\end{tabular}

Nota: Índice de masa corporal según curvas que por edad y sexo recoge la Fundación F. Orbegozo. DSM IV: peso inferior al $85 \%$ del esperable según edad y sexo. CIE 10: IMC igual o inferior a 17,5. 
Tabla 2. Medias en EAT y en BSQ según edad y sexo

\begin{tabular}{|c|c|c|c|c|c|c|}
\hline \multirow{2}{*}{ Edad } & & \multirow[b]{2}{*}{$n$} & \multicolumn{2}{|c|}{$E A T^{t}$} & \multicolumn{2}{|c|}{$B S Q^{2}$} \\
\hline & & & Media & $(D S)$ & Media & $(D S)$ \\
\hline \multirow[t]{2}{*}{14 años } & Hombre & 22 & 11,04 & $(5,8)$ & & \\
\hline & Mujer & 16 & 20,43 & $(17,14)$ & 80,44 & $(39,50)$ \\
\hline \multirow{2}{*}{15 años } & Hombre & 39 & 8,94 & $(5,28)$ & & \\
\hline & Mujer & 42 & 18,52 & $(14,65)$ & 88,05 & $(40,50)$ \\
\hline \multirow[t]{2}{*}{16 años } & Hombre & 45 & 10,53 & $(8,31)$ & & \\
\hline & Mujer & 49 & 26,57 & $(20,14)^{*}$ & 92,06 & $(42,79)$ \\
\hline \multirow[t]{2}{*}{17 años } & Hombre & 42 & 8,86 & $(3,73)$ & & \\
\hline & Mujer & 61 & 17,87 & $(11,8)$ & 90,41 & $(31,72)$ \\
\hline \multirow{2}{*}{18 años } & Hombre & 22 & 9,5 & $(3,72)$ & & \\
\hline & Mujer & 29 & 16,48 & $(10,93)$ & 82,64 & $(37,47)$ \\
\hline \multirow[t]{2}{*}{ TOTAL } & Hombre & 170 & 9,71 & $(5,84)^{* *}$ & & \\
\hline & Mujer & 197 & 20,17 & $(15,52)$ & 88,37 & $(37,85)$ \\
\hline
\end{tabular}

Nota: ${ }^{\star} \mathrm{p}<0,001$ con mujeres de 17 y 18 años; ${ }^{\star *} \mathrm{p}<0,001$ respecto a total mujer; ${ }^{1}$ cuestionario para mujeres y hombres; ${ }^{2}$ cuestionario para mujeres.

Tabla 3. Medias en EAT y en BSQ según la distribución por evaluación del estado nutricional

\begin{tabular}{ccccc}
\hline & \multicolumn{2}{c}{$E A T$} & \multicolumn{2}{c}{ MSQ $^{1}$} \\
& Media & (DS) & Media & (DS) \\
\hline Bajo peso & 14,90 & $(14,38)$ & 76,91 & $(44,38)$ \\
Normopeso & 14,71 & $(13,50)$ & 84,08 & $(36,00)$ \\
Sobrepeso & 17,46 & $(12,99)$ & 111,44 & $(30,76)^{\star}$ \\
Obesidad & 15,84 & $(11,12)$ & 91,80 & $(37,60)$ \\
\hline
\end{tabular}

Nota: ${ }^{*} \mathrm{p}<0,01$ respecto a bajo peso y normopeso.

tró una relación estadísticamente significativa en el grupo mujer, entre la puntuación en el BSQ y la puntuación en el EAT ( $p<0,0001)$, con un coeficiente de determinación $R^{2}$ de 0,43 . Distribuyendo a las mujeres en función del riesgo de padecer trastorno alimentario según su puntuación en el EAT, aquellas con dicho riesgo (EAT $\geq 30$ ) tuvieron una puntuación media significativamente mayor en el cuestionario BSQ [128,33 (DT $=32,08)]$ que la de los grupos con puntuaciones en el EAT entre 20 y 30 $[102,7(\mathrm{DT}=33,62)]$ y la del grupo con puntuación < 20 en el EAT, 71,95 $(28,54)$, con un valor $p<0,001$ en ambos casos. También existieron diferencias significativas en la puntuación BSQ entre las mujeres con puntuación EAT inferior a 20 y con EAT de 20 a 30 $(\mathrm{p}<0,001)$.
Relación entre variables antropométricas y las variables BSQ y EAT

Distribuyendo a los sujetos según los criterios de valoración de su estado nutricional, se encontró que en el BSQ, las mujeres con sobrepeso puntuaban significativamente más alto $(\mathrm{p}<0,01)$ que las de bajo peso y normopeso, no encontrándose diferencias significativas en la puntuación EAT siguiendo este mismo criterio ni entre las mujeres, ni en el total de alumnos (véase la Tabla 3).

Los sujetos con criterio antropométrico de anorexia según CIE 10 (IMC < 17,5), fueron 12 alumnos y no mostraron diferencias significativas en la puntuación en el EAT con respecto a los que no cumplían dicho criterio: $19,25(\mathrm{DT}=17,85)$ vs. 15,26 (DT 12,99 ). Tampoco existieron diferencias significativas en la puntuación del 
cuestionario BSQ entre las mujeres distribuidas según este mismo criterio: 56,5 $(\mathrm{DT}=76,88)$ vs. media 88,37 (DT $=37,75)$.

Descripción de la muestra según dietas, auto-valoración del volumen corporal y deseo de modificarlo

El porcentaje de mujeres que había seguido una dieta durante el último año fue de $22,84 \%$ y el de hombres $2,94 \%$ ( $p<0,001$ ). Un $61,96 \%$ de la muestra conocía familiares o amigos que hacían dieta, sin hallarse diferencias entre sexos.

Se auto-valoraron un poco, bastante $o$ muy obesos un $44,67 \%$ de las mujeres frente a un $20 \%$ de los hombres, con normopeso un $44,16 \%$ de las mujeres y un $55,88 \%$ de los hombres y con algún grado de delgadez se auto-valoraron un $11,17 \%$ de las mujeres y un $24,12 \%$ de los hombres. Desearon mayor delgadez un $70,05 \%$ de las mujeres frente a un 20 , $59 \%$ de los hombres.

\section{Auto-valoración del volumen corporal en relación al estado nutricional}

Se auto-valoran en algún grado obesos un $33,24 \%$ de los adolescentes, con normopeso un $49,59 \%$ y delgados un
$17,17 \%$. Al comparar la auto-valoración que del volumen corporal hacen los alumnos, con los datos objetivos proporcionados por el IMC, observamos que entre los sujetos con bajo peso un $28 \%$ de las mujeres se consideran un poco, bastante o muy obesas, frente a ninguno de los hombres. La mayoría de los sujetos con normopeso de ambos sexos se consideran normales, si bien un $30 \%$ de mujeres sobrevaloran su volumen corporal, mientras que un $28,6 \%$ de hombres lo infravaloran. Entre los sujetos con sobrepeso un $65 \%$ de los hombres frente a un $22 \%$ de las mujeres se consideran normales, siendo la valoración de las mujeres de esta categoría más acorde a su IMC objetivo. La auto-valoración de los sujetos obesos fue más acorde a su IMC, no observándose grandes variaciones entre los sexos, aún así se observa que un $22,9 \%$ de las mujeres se autovalora con un volumen normal (véase la Tabla 4).

Deseo de modificar el volumen corporal en función de la auto-valoración del mismo

Los sujetos de ambos sexos que se auto-valoran un poco, bastante, o muy obesos manifiestan su interés por estar

Tabla 4. Auto-valoración del volumen corporal en relación a la evaluación del estado nutricional según el IMC y el sexo

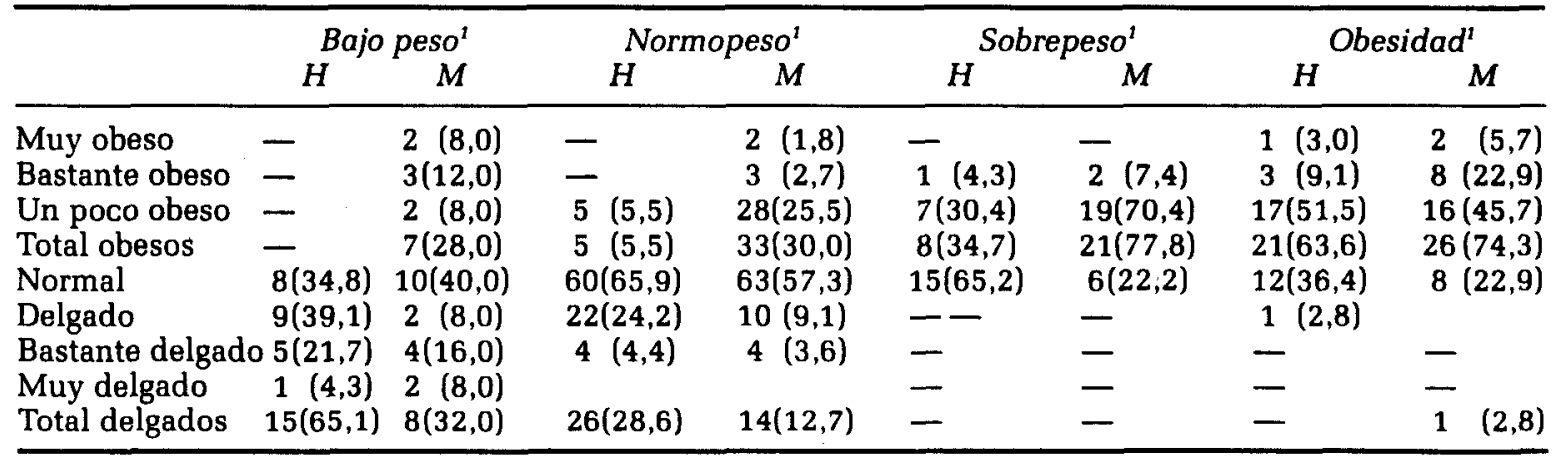

Nota: 'n (porcentaje sobre la columna); H: hombre, M: mujer. 
más delgados (porcentaje superior al $90 \%$ ). El $60,9 \%$ de las mujeres que se auto-valoran con normopeso desearían estar más delgadas y $37,9 \%$ igual, mientras que el $84,2 \%$ de los hombres de esta categoría desean continuar igual y sólo un $10,5 \%$ más delgados. Las mujeres que se auto-valoran delgadas o bastante delgadas desean mayoritariamente seguir igual $(84,6$ y $87,5 \%$ respectivamente), mientras que en los hombres se observó una tendencia a querer ganar peso entre los que se auto-valoran bastante delgados $(88,9 \%)$ y en menor medida entre los que se consideran simplemente delgados, $(45,2 \%)$.

\section{Medias en los cuestionarios EAT y BSQ en relación a la auto-valoración del volumen corporal}

La media en el EAT fue significativamente más alta para las mujeres que se auto-valoraron un poco obesas, normales y delgadas con respecto a los hombres con la misma auto-valoración. Las mujeres que se auto-valoraron bastante obesas obtuvieron una media en el EAT significativamente mayor que las que se auto-valoraron normales, delgadas o bastante delgadas. Las mujeres que se auto-valoraron como un poco obesas puntuaron significativamente más alto que las que lo hicieron como normales. No se observaron diferencias en la puntuación EAT entre los hombres en función de la auto-valoración del volumen corporal.

En el BSQ, las mujeres que se autovaloraron muy obesas tuvieron una puntuación media significativamente más alta que las que se consideraron normales, delgadas, bastante delgadas y muy delgadas. Igualmente, el BSQ es significativamente mayor para las mujeres que se auto-valoraron como bastante o un poco obesas, con respecto a las que lo hicieron como normales, delgadas o bastante delgadas (véase la Tabla 5).

Medias en los cuestionarios EAT y $B S Q$ según el deseo de modificar el volumen corporal y en relación a dietas

En ambos sexos, los sujetos que desearon estar más delgados tuvieron una puntuación en el EAT significativamente mayor [mujeres $24,23(\mathrm{DT}=16,44)$ y hombres $13,14(\mathrm{DT}=9,44)$ ], que los que desearon permanecer igual o más obesos,

Tabla 5. Puntuación en los cuestionarios EAT y BSQ

según la auto-valoración del volumen corporal y sexo

\begin{tabular}{|c|c|c|c|c|c|c|}
\hline & \multicolumn{4}{|c|}{$E A T^{1}$} & \multicolumn{2}{|c|}{$B S Q^{2}$} \\
\hline & \multicolumn{2}{|c|}{$H$} & \multicolumn{2}{|c|}{$M$} & \multirow{2}{*}{ Media } & \multirow{2}{*}{$(D S)$} \\
\hline & $\overline{\text { Media }}$ & (DS) & Media & $(D S)$ & & \\
\hline Muy obeso & & & 35,17 & $(20,15)$ & 150,83 & $(33,81)^{c}$ \\
\hline Bastante obeso & 13,75 & $(4,99)$ & 36,37 & $(21,50)^{a}$ & 120,56 & $(38,96)^{d}$ \\
\hline Un poco obeso & 11,55 & $(9,61)$ & 25,17 & $(16,70)^{\star b}$ & 107,25 & $(32,21)^{d}$ \\
\hline Normal & 9,61 & $(4,82)$ & 14,61 & $(9,01)^{\star}$ & 73,73 & $(51,77)$ \\
\hline Delgado & 7,48 & $(3,06)$ & 12,08 & $(10,01)^{\star *}$ & 51,77 & $(19,16)$ \\
\hline Bastante delgado & 9,89 & $(6,41)$ & 13,12 & $(10,26)$ & 46,87 & $(11,74)$ \\
\hline Muy delgado & & & 6,50 & $(0,7)$ & 61,00 & $(29,70)$ \\
\hline
\end{tabular}

Nota: ${ }^{1}$ Media (DS) H: hombre, M: mujer; ${ }^{2}$ Cuestionario para mujeres. ${ }^{*} \mathrm{p}<0,001$ entre hombres y mujeres; ${ }^{\star *} \mathrm{p}<0,05$ entre hombres y mujeres. ${ }^{a} \mathrm{p}<0,05$ entre mujeres con normal, delgado y bastante delgado. ${ }^{\mathrm{b}} \mathrm{p}<0,05$ entre las mujeres con normal. ${ }^{\mathrm{C}} \mathrm{p}<0,05$ con normal, delgado, bastante delgado y muy delgado. ${ }^{\mathrm{d}} \mathrm{p}<0,05$ con normal, delgado, bastante delgado. 
$\mathrm{p}<0,05$, [mujeres 11,31 (DT $=7,42)$ y $7,50(\mathrm{DT}=4,18)$, respectivamente, $\mathrm{y}$ hombres $8,94(\mathrm{DT}=3,92)$ y $8,18(\mathrm{DT}=$ 4,69), respectivamente].

Del mismo modo las mujeres que desearon estar más delgadas, obtuvieron una puntuación en el BSQ 103,47 (DT = 33,82), significativamente superior $\mathrm{p}<0,05$, a aquellas que desearon permanecer igual o más obesas $[53,91$ (DT $=17,76)$ y 47,17 (DT $=17,64)$ respectivamente].

Los sujetos que estaban haciendo, o habian hecho dieta durante el último año, puntuaron en el EAT significativamente más alto ( $p<0,001$ ), con respecto a los que no la hicieron $[34,72$ (DT = $18,43)$ vs. $12,29(\mathrm{DT}=8,82)]$.

Finalmente, la puntuación BSQ fue mayor en las mujeres que habían seguido dieta el último año $122,31(\mathrm{DT}=33,74)$ que en aquellas que no la hicieron 78,51 (DT $=32,91)$, con significación estadística $(p<0,001)$.

\section{Descripción del grupo con puntuación} en el EAT $>=30$ y sexo mujer

Este análisis se realizó exclusivamente entre el grupo de mujeres $(n=39)$ dado que el número de hombres que cumplieron este criterio fue de 3 y no podrían valorarse con rigor sus resultados.

Se observó que a la edad de 16 años la proporción de mujeres en riesgo de padecer trastorno alimentario era de $34,69 \%$, duplicándose o triplicándose respecto a los otros grupos de edad.

El porcentaje de mujeres con sobrepeso objetivo según criterios pediátricos, que puntuaron por encima de 30 en el EAT, fue de $25,93 \%$, con obesidad lo hizo un $20 \%$, con bajo peso un $20 \%$ y un $18,78 \%$ con normopeso.

Respecto a los sujetos que cumplían el criterio antropométrico de anorexia los porcentajes son escasamente valorables, dado el número reducido de población que cumple con ese criterio, 3,05\% de las mujeres y de estas sólo $2(1,02 \%)$ puntúan por encima de 30 en el EAT.

De las mujeres que se auto-valoraron como obesas en algún grado, un $36,78 \%$ puntuaron por encima de 30 , este porcentaje fue muy superior a las que se percibieron normales, $6,90 \%$, o delgadas un $4,35 \%$.

El $27,74 \%$ de las mujeres que deseaban estar más delgadas puntuaron por encima de 30 en el EAT, frente al 1,85\% de aquellas que desearían permanecer igual o el $0 \%$ de las que preferirían aumentar de peso.

En relación a las dietas se observó que un $60 \%$ de las mujeres que hacía o había hecho dieta personal en el último año puntuó por encima de 30 , así como el $25,93 \%$ de las que conocían un solo familiar y el $26,32 \%$ de las que conocían a varios de ellos (véase la Tabla 6).

\section{DISCUSIÓN}

\section{Prevalencia}

Los resultados del estudio muestran que el $19,8 \%$ de las mujeres y el $1,76 \%$ de los varones adolescentes, superan el punto de corte de 30 en el cuestionario EAT y, por tanto, son sujetos en riesgo de sufrir un trastorno del comportamiento alimentario. Estos resultados son similares a los encontrados, en población adolescente, en muestras valencianas (Villena et al., 1999; Toledo et al., 1999) y en muestras americanas (Pastore et al., 1996) e israelitas (Stein et al., 1997); y superiores a los hallados en muestras catalanas por Toro 89 (Toro et al., 1989), Canals 96 (Canals et al., 1996), en Suiza por Buddeberg 96 (Buddeberg-Fischer et al., 1996) y en Italia por Cotrufo 98 (Cotrufo et al., 1998). La ratio por sexos $(1 / 10)$ es común en la epidemiología de los trastornos alimen- 
200 Ana Sánchez, Felipe Hurtado, Julia Colomer, Silvia Saravia, José Monleón y Amparo Soriano

Tabla 6. Descripción del grupo con puntuación en el EAT $\geq 30$ y sexo mujer

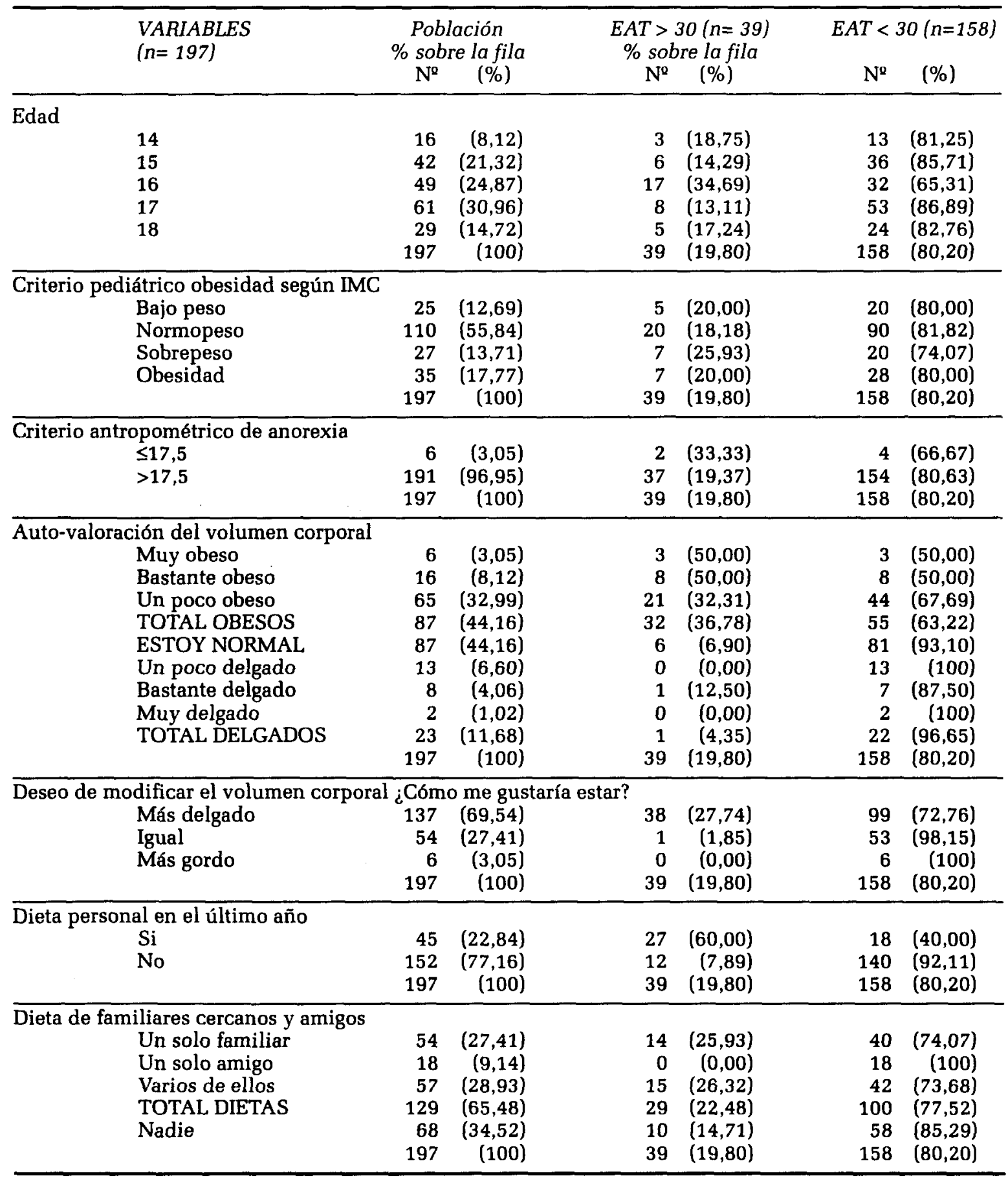

tarios, tanto anorexia como bulimia nerviosa (Toro, 1996). Aunque las cifras difieren, todos estos investigadores refieren en sus artículos que el riesgo de padecer un trastorno alimentario ha ido aumentando en los últimos años.
En los estudios revisados se encuentran diferentes metodologías para determinar la prevalencia de trastornos alimentarios, unos basan su información en los datos aportados exclusivamente por los test y medidas de autoinforme 
(Canals et al., 1996; Toro et al., 1989; Buddeberg-Fischer et al., 1996; Villena et al., 1999) (Toledo et al., 1999), mientras que otros utilizan estas medidas para la detección de sujetos en riesgo y posteriormente evalúan individualmente a los sujetos detectados mediante una entrevista personal, siguiendo los criterios diagnósticos internacionales de trastornos de la alimentación, DSM III-R, DSM IV o CIE 10 (Cotrufo et al., 1998).

Dado que el EAT es un cuestionario de autoinforme, la posibilidad de respuestas falso positivo o falso negativo debería considerarse a la hora de interpretar los resultados, siendo probablemente un factor a tener en cuenta en las divergencias en la prevalencia estimada por los diferentes estudios. Así, en aquellos en los que se realiza una entrevista individual para los sujetos en riesgo detectados con medidas de test o autoinforme, la población que cumple criterios diagnósticos de anorexia o bulimia es inferior (Cotrufo et al., 1998; Raich et al., 1992) a la de aquellos que se basan sólo en medidas de test o autoinformes y no realizan evaluación individual (Canals et al., 1996; Buddeberg-Fischer et al., 1996) 1-4 \% versus 8$20 \%$. No obstante la mayoría de los autores que se basan en test o autoinformes para estudiar la prevalencia de trastornos alimentarios, como en el presente estudio, utilizan en sus artículos los términos "actitudes alimentarias, síntomas de trastornos de la alimentación o riesgo de trastorno alimentario", en lugar de términos como «trastorno anoréxico, bulímico, o trastorno de la alimentación".

Entre las mujeres de 16 años, nuestros resultados detectan medias significativamente más altas y un porcentaje más elevado de mujeres en riesgo ( $E A T \geq 30$ ), respecto a las mujeres de los otros grupos de edad. Aunque la información epidemiológica coincide en situar la adolescencia como la edad de riesgo, otros estudios no encuentran un aumento señalado en una edad concreta dentro de la etapa adolescente. Sin embargo, en el cuestionario BSQ no se encontraron diferencias significativas entre los diferentes grupos de edad.

\section{Análisis descriptivo}

El porcentaje de sujetos con sobrepeso y obesidad que para cada edad y sexo se contemplan en las curvas de la Fundación Orbegozo (Hernández et al., 1988), fue significativamente mayor cuando se utilizaron percentiles de peso $(18,80 \%$ y $29,97 \%$ respectivamente) que cuando se usaron percentiles del IMC $(13,63 \%$ y 18,53 respectivamente). Los IMC utilizados para clasificar por sobrepeso u obesidad a los adultos son más elevados (2530 y superiores a 30 respectivamente), que los contemplados por edad y sexo en las curvas de la Fundación F. Orbegozo, en obesidad el percentil 90 más bajo corresponde a un IMC de 23,1 (hombres en el grupo de 14 años) y el más alto $26 ; 5$ (hombres de 17 años) y según el estado nutricional por encima del $120 \%$ de percentil 50, el IMC más bajo es de 23,41 hombres de 14 años y el más alto 26,52 (hombres de 17 años). Para el resto de análisis se decidió utilizar el IMC como ha sido recomendado recientemente por diversos autores (Dietz et al., 1998; Dalmau et al., 1999).

El porcentaje de sujetos que cumplían el criterio antropométrico de anorexia siguiendo los criterios DSM IV (peso inferior al $85 \%$ del esperable) no presentó diferencias significativas con los que cumplían con el criterio CIE 10 (IMC igual o inferior a 17,5). Por lo tanto, estos resultados apuntan a la necesidad de unificar criterios para el diagnóstico de problemas de obesidad y a la posibilidad de comparar resultados entre los diferentes estudios de anorexia, independientemente del uso de criterios CIE 10, que 
basa su medida en el IMC, o DSM IV que utiliza como criterio antropométrico el peso. No obstante y dado el tamaño muestral, serán necesarias nuevas investigaciones que apoyen estos resultados.

Los resultados de nuestro estudio muestran que un porcentaje superior al $30 \%$ de adolescentes presentan problemas de sobrepeso y obesidad tanto si se siguen los percentiles (percentil IMC 7590 para sobrepeso, percentil IMC superior a 90 para obesidad), (Dalmau et al., 1999; Himes et al., 1994), como siguiendo los criterios de valoración del estado nutricional (sobrepeso IMC 110-120\%, obesidad superior a $120 \%$ del percentil 50 para su sexo y edad) (Pombo, 1990). Estos resultados se aproximan a los encontrados en un estudio sobre prevalencia de obesidad en la Comunidad Valenciana realizado por Quiles en 1996 (Quiles et al., 1996a), que para el grupo de edad de 15 a 24 años encontró un $15,7 \%$ con sobrepeso (IMC 25-29) y un $3,7 \%$ con obesidad (IMC superior a 30 ), y difiere de los datos de Gutiérrez (Gutierrez et al., 1994) sobre población española, que encontró una prevalencia del $1 \%$ de obesidad (IMC mayor de 30) en el grupo de 20 a 24 años; así mismo, en la Comunidad Valenciana Toledo (Toledo et al., 1999) halló una prevalencia de sobrepeso (IMC $26-30$ ) del $7,33 \%$ y del $1,10 \%$ de obesidad leve (IMC $31-40$ ) en edades comprendidas entre los 14-21 años. Es probable que las diferencias puedan explicarse, entre otras razones, por las diferentes metodologías utilizadas. Mientras que unos utilizan la medición directa del peso y la altura para hallar el IMC (Quiles et al., 1996a) otros recogen las medidas antropométricas a partir de autoinformes (Gutierrez et al., 1994). Se ha cuestionado la validez de los estudios que estiman la prevalencia de la obesidad a partir de los datos declarados en autoinformes, por la tendencia observada en los sujetos a infra- valorar el peso y sobreestimar la talla declarada, por lo que se subestimaría la prevalencia de obesidad (Quiles et al., 1996b). Otra posible explicación de la mayor prevalencia de sobrepeso y obesidad en nuestro estudio, sería que los IMC son más bajos para su detección siguiendo las curvas por edad y sexo de la Fundación Orbegozo que diagnosticando según los criterios definidos para los adultos.

En cuanto a la auto-valoración del volumen corporal en relación a la valoración objetiva del estado nutricional, destaca la tendencia general de las mujeres a sobreestimar su volumen y la de los hombres a infravalorarlo, no obstante se observa una concordancia importante entre el obtenido y el auto-valorado (Tabla 4) en las mujeres con sobrepeso $(77,8 \%)$ y obesidad $(74,3 \%)$. Las mujeres con normopeso se consideran normales $(57,3 \%)$ o un poco obesas $(25,5 \%)$ y las de bajo peso se auto-valoran o normales $(40 \%)$ o delgadas $(34 \%)$. Drewnowski (Drewnowski et al., 1994) observó que en las mujeres existía el deseo de disminuir el peso, mientras que los hombres deseaban aumentarlo probablemente por su interés en ganar fuerza y desarrollo muscular.

Respecto al deseo de modificar el volumen corporal en función de la autovaloración del mismo, vuelven a observarse las discrepancias entre los sexos. Mayoritariamente las mujeres desearon estar más delgadas aunque se auto-valoraran normales (desearon de este grupo mayor delgadez un $60,9 \%$ ) o con problemas de peso (superior al $93 \%$ ) y las que se describieron delgadas prefirieron continuar mayoritariamente así (superior al $80 \%$ ). En los hombres que se autovaloraron normales, sólo un 10,5\% deseó estar más delgado. Ambos sexos coincidieron en su interés por perder peso cuando se auto-valoraron un poco, bastante o muy obesos. 
Estos datos constatan la presión social que en el mundo occidental se ejerce sobre la mujer, idealizando la delgadez y animándola a mantener cuerpos cada vez más delgados. Esta observación coincide con la bibliografía revisada para esta investigación (Fairburn et al., 1999).

Habían realizado dieta durante el último año un $13,62 \%$ de los sujetos, de los que el $90 \%$ fueron mujeres; mientras que un $60 \%$ de los alumnos conocía a varios familiares o amigos que realizaban dieta. Estos valores son similares a los encontrados por Toro (Toro et al., 1989) $(9,4 \%$ de adolescentes realizaban dieta y $79,78 \%$ conocían a personas cercanas que hacían dieta). Fairburn (Fairburn et al., 1997) encontró que el seguimiento de dietas por el sujeto o por sus padres, era uno de los factores de riesgo de bulimia nerviosa, y Edmunds encontró que los padres tenían un rol activo en las dietas de sus hijos (Edmunds et al., 1999).

\section{Correlación de variables}

Las puntuaciones en el EAT mostraron una relación estadísticamente significativa con las puntuaciones en el cuestionario para la evaluación de la imagen corporal BSQ, observándose medias significativamente más altas en el BSQ entre los sujetos con puntuaciones superiores a 30 en el EAT. Los resultados coinciden con los aportados por los autores del BSQ (Cooper et al., 1987).

No se hallaron diferencias estadísticamente significativas en el EAT en función de la distribución del estado nutricional, aunque la puntuación fue algo más elevada en el grupo con sobrepeso. Toro (Toro et al., 1989) encuentra puntuaciones significativamente más altas en el EAT $(p<0,05)$ entre los sujetos obesos y con sobrepeso, respecto a los sujetos normales o bastante delgados.
Las medias en el cuestionario BSQ fueron significativamente más altas en las adolescentes con sobrepeso (111,44 DS $30,76)$ respecto a las del grupo con bajo peso $(76,91$ DS 44,38$)$ y normopeso $(84,08$ DS 36).

El hecho de que las puntuaciones medias en el BSQ sean similares para todos los grupos de edad, a diferencia del EAT, y que sus puntuaciones suban cuando la persona presenta, según criterios objetivos, un exceso de peso, hace sospechar que si existe insatisfacción con la imagen corporal esta probablemente no se refiera a una distorsión perceptiva. En este sentido Rosen (Rosen et al., 1996) en su estudio de fiabilidad y validez del BSQ observó las puntuaciones más altas en el cuestionario, en las mujeres con problemas de obesidad (media $\mathbf{1 3 5 , 6 )}$ ), en la misma línea un estudio de metaanálisis realizado por Cash evaluando parámetros de imagen corporal entre mujeres con anorexia o bulimia nerviosa (Cash et al., 1997) informa que la insatisfacción corporal actitudinal es mayor y más significativa que los índices de distorsión perceptiva, y Mora (Mora et al., 1993) en una revisión de 24 artículos que evalúan las alteraciones perceptivas de la imagen corporal, señala que la mayoría de estudios no consigue demostrar que las anoréxicas sobreestimen su tamaño corporal en mayor medida que las personas sin trastorno alimentario.

Esta idea de que la insatisfacción con la imagen corporal no se debe a una distorsión perceptiva concuerda con nuestros resultados, en los que destaca el alto porcentaje de mujeres que auto-valorándose con un peso normal desearían estar más delgadas $(60,9 \%)$ y el que las mujeres que objetivamente presentan sobrepeso obtengan puntuaciones significativamente superiores en el BSQ respecto a las del grupo con peso normal o bajo peso.

Las medias en el cuestionario EAT han sido significativamente más altas en las 
adolescentes que se auto-valoran con exceso de peso, respecto a las que se perciben como normales o delgadas, este mismo resultado se observa en las puntuaciones del BSQ. En la misma dirección, de las 39 adolescentes con puntuación igual o superior a 30 en el EAT, 32 $(82,05 \%)$, se auto-valoran con exceso de peso. Toro (Toro et al., 1989) encontró igualmente esas diferencias.

Las puntuaciones obtenidas por los sujetos en los cuestionarios EAT y BSQ, fueron significativamente más altas entre aquellos que desearían estar más delgados, respecto a aquellos que preferían permanecer igual o más obesos. Del grupo de mujeres con puntuación igual o superior a 30 el EAT, $38(97,43 \%)$ pertenecen al grupo de los que desearían mayor delgadez, datos que concuerdan con los hallados por Toro (Toro et al., 1989).

El haber seguido una dieta restrictiva durante el último año marcó puntuaciones significativamente más altas en el EAT (media 34,72 DS18,93) con respecto a los que no la siguieron (media 12,29 DS8,82), y en la misma línea, de las 39 mujeres con puntuaciones de riesgo en EAT, 27 mujeres $(69,23 \%)$ habían limitado o estaban limitando su ingesta. La dieta restrictiva como factor de riesgo en el desarrollo de los trastornos alimentarios ha sido comunicada por diferentes investigadores (Patton et al., 1999). También, la puntuación en el cuestionario BSQ fue significativamente superior en las mujeres que hicieron dieta que en las que no la hicieron.

Los alumnos que conocían a varios familiares $y / 0$ amigos realizando dietas, puntuaron significativamente más alto que los que no conocían a nadie o sólo tenían a un amigo realizándola. En el cuestionario BSQ, aquellos que conocían a varios, puntuaron significativamente más alto que los que no conocían a nadie llevando a cabo una dieta.

\section{CONCLUSIONES}

La prevalencia de sujetos en riesgo de sufrir un trastorno de la alimentación $($ EAT $>30)$ en nuestra muestra fue del $19,8 \%$ en las mujeres y del $1,76 \%$ en los hombres. Las mujeres que se auto-valoraron como bastante o muy obesas y aquellas que habían realizado dieta en el último año obtuvieron una puntuación media por encima de 30 en el EAT.

Sin superar el punto de corte 30 se observaron puntuaciones significativamente más altas entre los sujetos que deseaban estar más delgados, respecto a los que deseaban permanecer igual o ganar peso. Las mujeres que se auto-valoraron un poco obesas también puntuaron significativamente más alto que las que se auto-valoraron con un peso normal.

En la distribución según el estado nutricional no se hallaron diferencias significativas en las puntuaciones en el EAT, aunque las medias fueron más elevadas para el grupo con sobrepeso y obesidad.

La prevalencia de sujetos que cumplían el criterio antropométrico de anorexia fue del $3,27 \%$ ( 12 alumnos), puntuando por encima de 30 en el EAT sólo 2 sujetos $(0,54 \%)$. Sin embargo, las puntuaciones en el EAT fueron superiores en los grupos con sobrepeso y obesidad, aunque sin superar el punto de corte de riesgo de 30 y sin significación estadística respecto a los otros grupos.

Para poder comparar la prevalencia de sobrepeso y obesidad es conveniente que en las investigaciones se unifiquen criterios tanto metodológicos (IMC vs. peso, curvas de desarrollo y crecimiento específica de infancia y adolescencia vs. criterios de obesidad para adultos) como en la recogida de información (peso y talla informado por el sujeto vs. medidos).

La distorsión de la imagen corporal medida a través del BSQ fue significativamente superior en las mujeres con sobrepeso, así como en aquellas que se 
auto-valoraron con exceso de peso, en las que deseaban mayor delgadez y en las que realizaron dieta en el último año.

Sería conveniente realizar investigaciones que valoren si la distorsión de la imagen corporal es patognomónica de los trastornos alimentarios o es una característica propia de la etapa adolescente, más acentuada por ser un periodo en el que la autoestima está altamente ligada a la imagen física y por situarnos en una sociedad que promueve como canon de belleza la extrema delgadez. Si esto es así, los programas preventivos deberían probablemente dirigir sus esfuerzos hacia las actitudes y cogniciones que sobre la imagen corporal genera e impone la "cultura de la delgadez» y no a actuar sobre una supuesta distorsión perceptiva.

Un programa preventivo para nuestra área de salud debería contemplar los trastornos alimentarios desde una perspectiva global, sin olvidar la alta prevalencia de adolescentes con sobrepeso y obesidad, así como los factores relacionados con estos problemas, tanto nutricionales como ambientales, psicológicos y sociales.

\section{REFERENCIAS BIBLIOGRÁFICAS}

American Psychiatric Association (1994) Diagnostic and Statiscal Manual of Mental Disorder ( $4^{a}$ ed.) Washington (Edición española, Barcelona: Masson, 1995).

Buddeberg-Fischer, B., Bernet, R., Sieber, M., Schmid, J., y Buddeberg, C. (1996). Epidemiology of eating behaviour and weight distribution in 14- to 19-year-old Swiss students. Acta Psychiatrica Scandinavica, 93, 296-304.

Calvo, R. (1985). La anorexia nerviosa. En J.A. Carrobles (Ed.), Análisis y Modificación de Conducta II (pp. 71-89). Madrid: UNED.

Canals, J., Carbajo, G., Fernández, J., MartiHenneberg, C., y Domenech, E. (1996). Biopsychopathologic risk profile of adolescents with eating disorder symptoms. Adolescence, 31, 443-450.
Cash, T.F., y Deagle, E.A. (1997). The nature and extent of body-image disturbances in anorexia nervosa and bulimia nervosa: a meta-analysis. International Journal of Eating Disorders, 22, 107-125.

Castro, J., Toro, J., Salamero, M., y Guimera, E. (1991). El test de actitudes alimentarias: validación de la versión española. Evaluación Psicológica, 2, 175-190.

Cooper, P.J., Taylor, M.J., Cooper, Z., y Fairburn, C.G. (1987). The development and validation of the BodyShape Questionnaire. International Journal of Eating Disorders., 6, 485-494.

Cotrufo, J., Barretta, V., y Monteleone, P. (1998). Un estudio epidemiológico sobre los trastornos de la conducta alimentaria en dos institutos de segunda enseñanza en Nápoles. International Journal of Eating Disorders, 12, 86-89.

Dalmau, J., y Fernández, J. (1999). Obesidad infantil y juvenil. Pediatrika, Suplemento 1, 94-101.

Dietz, W.H., y Robinson, T.N. (1998). Use of the body mass index (BMI) as a measure of overweight in children and adolescents [editorial; comment]. Journal of Pediatrics, 132, 191-193.

Drewnowski, A., Kurth, C.L., y Krahn, D.D. (1994). Body weight and dieting in adolescence: impact of socioeconomic status. International Journal of Eating Disorders, 16, 61-65.

Edmunds, H., y Hill, A.J. (1999). Dieting and the family context of eating in young adolescent children. International Journal of Eating Disorders, 25, 435-440.

Fairburn, C.G., Cooper, Z., Doll, H.A., y Welch, S.L. (1999). Risk factors for anorexia nervosa: three integrated case-control comparisons. Archives of General Psychiatry, 56, 468-476.

Fairburn, C.G., Welch, S.L., Doll, H.A., Davies, B.A., y O'Connor, M.E. (1997). Risk factors for bulimia nervosa. A communitybased case-control study. Archives of General Psychiatry, 54,509-517.

Fisher, M., Pastore, D., Schneider, M., Pegler, C., y Napolitano, B. (1994). Eating attitudes in urban and suburban adolescents. International Journal of Eating Disorders. 
Gard, M.C., y Freeman, C.P. (1996). The dismantling of a myth: a review of eating disorders and socioeconomic status. International Journal of Eating Disorders, 20, 1-12.

Garner, D.M., y Garfinkel, P.E. (1979). The Eating Attitudes Test: an index of the symptoms of anorexia nervosa. Psychological Medicine, 9, 273-279.

Gutiérrez, J.L., Regidor, E., y Rodríguez, E. (1994). Prevalencia de la obesidad en España. Medicina Clínica, 102, 10-13.

Hernández, M., Castellet, J., y Narvaiza, J.L. (1988). Curvas y tablas de crecimiento. Madrid: Instituto de Investigaciones sobre Crecimiento y Desarrollo. Fundación F. Orbegozo. Garsi Editorial.

Himes, J.H., y Dietz, W.H. (1994). Guidelines for overweight in adolescent preventive services: recommendations from an expert committee. The Expert Committee on Clinical Guidelines for Overweight in Adolescent Preventive Services. American Journal of Clinical Nutrition., 59, 307-316.

McLaren, D.S., y Read, W.W. (1972). Classification of nutritional status in early childhood. Lancet, 2, 146-148.

Mora, M., y Raich, R.M. (1993). Prevalencia de las alteraciones de la imagen corporal en poblaciones con trastorno alimentario. Revista de Psiquiatría de la Facultad de Medicina de Barcelona, 3, 113-131.

Pastore, D.R., Fisher, M., y Friedman, S.B. (1996). Abnormalities in weight status, eating attitudes, and eating behaviors among urban high school students: correlations with self-esteem and anxiety. Journal of Adolescent Health, 18, 312-319.

Patton, G.C., Selzer, R., Coffey, C., Carlin, J.B., y Wolfe, R. (1999). Onset of adolescent eating disorders: population based cohort study over 3 years. British Medical Journal, 318, 765-768.

Pombo, M. (1990). Etiología, valoración y tratamiento de la obesidad en el niño. En M. Pombo (Ed.), Endocrinología Pediátrica. (pp. 219-233). Madrid: Díaz de Santos.

Quiles, J., y Vioque, J. (1996a). Prevalencia de obesidad en la Comunidad Valenciana. Medicina Clínica, 106, 529-533.

Quiles, J., y Vioque, J. (1996b). Validez de los datos antropométricos declarados para la determinación de la prevalencia de obesidad. Medicina Clínica, 106, 725-729.

Raich, R.M., Rosen, J.C., Deus, J., Pérez, O., Requena, A., y Gross, J. (1992). Eating disorder symptoms among adolescents in the United States and Spain: A comparative study. International Journal of Eating Disorders, 11, 63-72.

Rosen, J.C., Jones, A., Ramírez, E., y Waxman, S. (1996). Body Shape Questionnaire: studies of validity and reliability. International Journal of Eating Disorders, 20, 315319.

Stein, D., Meged, S., Bar-Hanin, T., Blank, S., Elizur, A., y Weizman, A. (1997). Partial eating disorders in a community sample of female adolescents. Journal of the American Academy of Child and Adolescent, 36, 1116-1123.

Toledo, M., Ferrero, J., Capote, J., Roger, L., Sánchez, M., Donet, E., y Tormo, E. (1999). Incidencia de comportamientos anoréxicos y bulímicos en la Comunidad Valenciana. Cuadernos de Medicina Psicosomática y Psiquiatría de Enlace, (51-52), 38-48.

Toro, J. (1996). El cuerpo como delito. Barcelona: Ariel.

Toro, J., Castro, J., García, M., Pérez, P., y Cuesta, L. (1989). Eating attitudes, sociodemographic factors and body shape evaluation in adolescence. British Journal of Medical Psychology, 62(Pt 1), 61-70.

Toro, J., Salamero, M., y Martínez, E. (1994). Assessment of sociocultural influences on the aesthetic body shape model in anorexia nervosa. Acta Psychiatrica Scandinavica., 89, 147-151.

Toro, J., y Vilardell, E. (1987). Anorexia nerviosa. Barcelona: Martínez Roca.

Villena, J., y Castillo, M. (1999). Intervención preventiva de los trastornos de la conducta alimentaria en mujeres adolescentes. Programa "Tal como eres». Valencia: Generalitat Valenciana. Consellería de Sanitat. Dirección General de Salud Pública. Serie $\mathrm{D} \mathrm{n}^{0} 28$.

World Health Organization. (1993). The ICD10 Classification of Mental and Behavioural Disorders. Diagnostic Criteria for Research. Geneva: WHO. 